Resiliencia como estrategia para la práctica de enfermería. Una propuesta teórica.

\title{
Autores:
}

Fabiola Arriagada Gallardo. Enfermera, Magister en Enfermería. Docente Facultad de Enfermería Universidad de Concepción, Chile. E-mail: faarriagada@udec.cl

Patricia Jara Concha. Enfermera, Doctora en Enfermería. Docente Facultad de Enfermería Universidad de Concepción, Chile. E mail: pjara@udec.cl

\section{> Resumen}

La transición del estado de salud a enfermedad es un proceso complejo que afecta a una persona, quien responde positiva o negativamente frente a esta adversidad. La resiliencia hace que algunos respondan de una manera positiva, saliendo incluso fortalecidos de una dificil situación, actuando como potenciador de conductas que favorecen el restablecimiento de la salud o estabilización de enfermedades crónicas, convirtiéndose en un aliado en la práctica de enfermería. Este articulo tiene como objetivo presentar una propuesta teórica basada en la Teoría de rango medio de Afaf Meleis analizando transición y en la Teoría de la promoción de la salud de Nola Pender identificando factores protectores de resiliencia para intervenciones futuras.

> Palabras claves: Resiliencia psicológica; enfermería; promoción de la salud. 

practice. A theoretical proposal.

\section{> Abstract}

The transition from health to disease is a complex process that affects a person who responds positively or negatively to this adversity. Resilience makes some people respond in a positive way, furthermore, they are able to emerge stronger from a difficult situation, also they act as an enhancer of behaviors promoting health restoration or stabilization of chronic diseases. Finally they become an ally of nursing practice. The claim of this article is a theoretical proposal based on Afaf Meleis' midrange theory in order to analyze the process of transition; and Nola Pender's theory of health promotion, to identify protective factors of resilience for future interventions.

, Keywords: Resilience, psychological; nursing; health promotion (DeCS). 


\section{) Introducción}

Durante el desarrollo de la vida profesional, los/ as enfermeros/as se ven enfrentados a diversas situaciones, en donde la persona no solo ha perdido su condición de salud, sino que también se ve expuesta a distintos factores que desestabilizan su estado inicial, planteándose un escenario de transición. En este contexto, como variadas son las personas, variadas también son sus respuestas.

Resiliencia es una respuesta saludable frente a la adversidad que desarrollan las personas al sobreponerse a las condiciones difíciles, superarlas y salir fortalecidos de ellas o incluso transformados, considerándose una capacidad humana trascendental para enfrentar exitosamente los diversos desafíos que presenta la vida (1).

Indagar sobre cuáles son los factores que afectan el nivel de resiliencia de las personas entrega herramientas para comprender las respuestas que se presentan frente a un estado de inestabilidad en su salud, mejorando las estrategias de intervención sin apoyarse solo en las necesidades y la enfermedad, sino que también direccionando a un modelo de prevención y promoción, basado en las potencialidades, los recursos y la capacidad de sobreponerse a la adversidad que el ser humano tiene en sí mismo y a su alrededor (2).

Detectar y debilitar los factores amenazantes de la resiliencia en el cuidador o en el familiar encargado de una persona enferma, permitirá en la práctica de enfermería obtener una mejor respuesta frente a la adversidad, haciéndolo participe en el proceso, mejorando la adherencia al tratamiento, disminuyendo los reingresos hospitalarios y las interconsultas innecesarias, por ende, salir fortalecido después de la crisis que ha provocado una enfermedad aguda.
Promover la resiliencia es un llamado a relacionarse con el usuario en su totalidad(3), en cada instancia de cuidado que proporciona la enfermera/o.

\section{, Resiliencia}

Resiliencia es un concepto relativamente nuevo que ha sido incluido como parte de los programas de promoción de la salud, aunque existe desde siempre en la humanidad. Este concepto fue incorporado desde la física y se utiliza en este campo para referirse a la capacidad que tienen los materiales para resistir choques imprevistos sin quebrarse, o a recobrar su forma original después de haber sido sometidos a golpes o altas presiones (4). En las ciencias sociales se introduce en los años setenta en un estudio a un grupo de personas desde el momento de su nacimiento hasta los cuarenta años, percibiéndose que algunos niños con factores de riesgo eran capaces de salir adelante y ser exitosos en la vida y constituir familias estables (5). Así se comienza a integrar el concepto de resiliencia no solo a un individuo, sino también a la familia y comunidad.

Existen variadas definiciones de resiliencia, entre ellos "la capacidad humana para enfrentar, sobreponerse, ser fortalecido o transformar activamente la realidad, habiendo vivido experiencias de adversidad" (6), "cualidad de las personas para resistir y adaptarse a situaciones traumáticas y dificiles" y, también, se ha definido como la "capacidad de una persona o grupo para seguir proyectándose en el futuro a pesar de acontecimientos desestabilizadores, condiciones de vida dificiles y de traumas graves" (8). Si bien son múltiples las definiciones de resiliencia, la gran mayoría presenta tres componentes que son esenciales: la noción de amenaza o adversidad, adaptación positiva y el proceso que incluye factores individuales, cognitivos emocionales y culturales del individuo. 
La resiliencia no es total, absoluta o lograda para siempre, si no que es un proceso que depende de la experiencia traumática vivida que puede superar los recursos del individuo y que varía según circunstancias, contexto, etapa de la vida y entorno cultural (9). Ser resiliente no significa una protección absoluta, tiene límites y no hay persona que sea invulnerable, por lo que juegan un papel importante los factores de riesgo y los factores de protección (10).

En el momento en que la persona se ve expuesta a una situación estresante, surge resiliencia de la interacción de los factores de riesgo y los factores de protección, los cuales se manifiestan en un determinado contexto biológico y sociocultural, evidenciando la capacidad de salir adelante y de obtener un beneficio para su vida a partir de aquella experiencia. Se debe reconocer que los factores protectores y de riesgo no son estáticos, sino que varían de una persona a otra, considerando elementos como género y edad, incluso según la etapa del ciclo vital y el medio en que está inserta.

Los factores de riesgo pueden ser biológicos como, por ejemplo, bajo peso al nacer, defectos congénitos, etc., y también socio-ambientales como la pobreza, baja escolaridad, entre otros. Hablar de factores de riesgo podría evidenciar un escenario negativo, pero es importante conocer estas condiciones para trabajar sobre ellas y evitar su efecto (11).

Por otro lado, los factores protectores son herramientas utilizables en contra de la adversidad que provienen del mismo individuo o de su medio, llamados también pilares de la resiliencia que se exponen a continuación (12):

Autoestima consistente: es la base de los demás pilares y es el fruto del cuidado afectivo consecuente del niño o adolescente por un adulto sig- nificativo, "suficientemente" bueno y capaz de dar una respuesta sensible.

Introspección: es el arte de preguntarse a sí mismo y de darse una respuesta honesta.

Independencia: se define como el saber fijar límites entre uno mismo y el medio con problemas; la capacidad de mantener distancia emocional y física sin caer en el aislamiento.

Capacidad de relacionarse: es decir, la habilidad para establecer lazos e intimidad con otras personas.

Iniciativa: el gusto de exigirse y ponerse a prueba en tareas progresivamente más exigentes. La capacidad para planear e iniciar tareas con la confianza y esperanza de lograr los objetivos propuestos.

Humor: la capacidad de reír y encontrar lo cómico en la propia tragedia.

Creatividad: la capacidad de crear orden, belleza y finalidad a partir del caos y del desorden.

Moralidad: entendida como la consecuencia para extender el deseo personal de bienestar a todos los semejantes y la capacidad de comprometerse con valores.

Capacidad de pensamiento crítico: permite analizar críticamente las causas y responsabilidades de la adversidad que se sufre.

Los pilares de la resiliencia expuestos son factores que debieran formar parte de un instrumento de valoración de los/as enfermeros/as en las distintas unidades y servicios donde se ejerce la práctica, con el fin de detectarlos para la obtención de respuestas resilientes, ya sea en condición de enfermedad o en la potencialización de conductas de promoción de la salud. 
Junto con los pilares de resiliencia, se requiere de la relación dinámica de fuentes interactivas, como lo plantea Grotberg, que se visualizan en las expresiones verbales de las personas con características resilientes (13) y que dan como respuestas comportamientos saludables frente a eventos traumáticos. Estos son:

Relacionados con el apoyo que la persona puede percibir, "yo tengo".

Relacionadas con las fortalezas intrasíquicas y condiciones internas de las personas "yo estoy" y "yo soy".

Relacionadas con las habilidades de la persona para relacionarse y resolver problemas, "yo puedo".

Los/as enfermeros/as deben estar capacitados desde su formación de pregrado para detectar estas fuentes interactivas y reconocer estas expresiones en las personas, utilizarlas como apoyo fundamental para incrementar respuestas saludables frente a la adversidad.

La resiliencia también ha sido estudiada en Chile por un investigador que plantea un Modelo ecológico interaccional, basado en lo planteado por Grotberg, desde donde surge un instrumento de medición del nivel de resiliencia SV-RES, compuesto por 12 factores que son: Identidad, Autonomía, Satisfacción, Pragmatismo, Vínculos, Redes, Modelos, Metas, Afectividad, Autoeficacia, Aprendizaje y Generatividad $(4,14)$.

Estos doce factores de resiliencia son los aspectos donde los/as enfermeros/as pueden intervenir para incrementar la capacidad de salir adelante en la adversidad a personas y familiares que están dependientes de su cuidado, factores modificables con la gestión de enfermería dirigidos a construir y promocionar la resiliencia.

\section{) Transición y resiliencia}

La teoría de la Transición de Afaf Meleis, permite comprender el proceso que se presenta cuando se enfrentan episodios, ya sean situacionales o de transformación de salud a enfermedad (15). Ambos conceptos, resiliencia y transición, se relacionan estrechamente, ya que ambos plantean factores de riesgo o de inhibición y factores facilitadores o de protección que se entrelazan para el desarrollo del proceso de crisis que se evidencia. Un alto nivel de resiliencia sugiere que la persona posee ciertas condiciones que le permiten vivenciar un estado de transición en forma positiva, terminando fortalecida y con nuevas herramientas que podrá utilizar en situaciones de adversidad a futuro.

El/la enfermero/a debe lograr una comunicación con la persona y su familia que les permita reconocer el proceso de transición y los factores que influyen en su nivel de resiliencia, es decir, que mientras mayor sea el reconocimiento por parte de la persona/famlia, mejor será la forma en que evoluciona el proceso de transición enfermedad hacia el equilibrio de su condición de salud.

La promoción de la resiliencia hace que el proceso de transición se desarrolle de una manera adecua$\mathrm{da}$, entregando nuevas herramientas a la persona y a su entorno para enfrentar nuevas situaciones de crisis y desarrollar transiciones saludables.

\section{Promoción de la salud y resiliencia}

Establecer una relación entre resiliencia y promoción de la salud, de acuerdo al modelo de Nola Pender, da importancia a los procesos cognitivos, al cambio de conducta y autocreencias, como la autoatribución, autoevaluación y autoeficacia (16). 
Este modelo ilustra la variada naturaleza de las personas que interactúan con el entorno, intentando alcanzar un nivel de salud óptimo y como las experiencias individuales afectan las acciones en salud. Así, una conducta previa en relación a salud, unida a factores personales biológicos, psicológicos y socioculturales, influyen directamente en cómo la persona percibe las barreras o beneficios, frente a acciones que conllevan a conductas de salud eficientes. La autoeficacia percibida, que es la capacidad de enfrentar las barreras que se interponen a una vida saludable y ejecutar una conducta promotora de salud, depende directamente de influencias interpersonales y situacionales previas vividas. Una conducta promotora de salud es el resultado de una acción de salud positiva, como el bienestar óptimo, el cumplimiento personal y la vida productiva (17).

Algunos de los principales supuestos de esta teoría, se orientan estimular que la persona se enfoque en expresar su propio potencial de la salud humana y valorar el crecimiento en las direcciones observadas como positivas y, el intento de conseguir un equilibrio entre el cambio y la estabilidad (18).

Las personas interactúan con el entorno teniendo en cuenta su complejidad biosicosocial, transformándolo con el paso del tiempo. El/la enfermero/a forma parte de este entorno, ejerciendo influencia en las personas a lo largo de su vida. Entre las afirmaciones teóricas de este modelo, destaca que la conducta previa, las características heredadas y adquiridas influyen en las creencias, el afecto y la promulgación de las conductas de promoción de la salud (19). Cuando las emociones y el afecto se asocian con una conducta, aumenta la probabilidad de compromiso y acción. Las familias y los cuidadores de salud son fuentes importantes de influencia que pueden hacer aumentar o dis- minuir el compromiso para adoptar una conducta promotora de salud (20).

En este modelo, se evidencia la importancia atribuida al entorno y a la familia del afectado, en lo que se refiere a conductas que estimulan y fomentan prácticas que fortalecen la salud y preparan el entorno propicio para la estabilización.

Para cualquier persona y su familia, la inestabilidad en su salud supone una carga emocional y es vital aliviar sus dudas y apoyarlos en el proceso de transición que se evidencia (21). Resiliencia es una respuesta que depende de la visión de cada persona, de elementos afectivos y cognitivos proactivos frente a los problemas y el camino que se recorre para lograr la acción en la transición vivida, finalizando con una respuesta que se asocia directamente a la obtención de un modelo de salud positivo. Es así que la resiliencia incrementa la capacidad del individuo de adquirir conductas de salud adecuadas, ya que se dispone a ver más allá de la transición que vive, logrando un producto final positivo y de crecimiento personal. El tener la capacidad de enfrentar la adversidad de una manera óptima, con alto nivel de resiliencia, permitirá evolucionar de la tristeza y el desconcierto hacia un escenario en donde la adversidad es un camino de crecimiento (22).

\section{> Propuesta teórica}

Conceptos como transición, promoción de la salud y resilencia interactúan con la finalidad de obtener respuestas positivas frente a episodios de adversidad, lo cual puede ser potenciado a través de la práctica de enfermería.

El paso de la salud a la enfermedad es una adversidad que se presenta como proceso de transición que afecta a la persona y a su familia, los cuales responden de variadas formas dependiendo de 
factores ambientales, personales, socioculturales en que han estado insertos y han adquirido como experiencias de vida, factores que pueden resultar protectores o de riesgo. Las respuestas de las personas pueden ser variadas y múltiples, enfrentando la adversidad como una propuesta al crecimiento personal, promotoras de salud, confluyendo en la resolución de su problema de inestabilidad (23).

Las influencias situacionales del entorno pueden generar el aumento o la disminución del compromiso o de la participación en el proceso de transición y en la obtención de una conducta promotora de salud, por lo que la intervención de enfermería es vital para el desarrollo de una transición eficiente, aumentando el nivel de resiliencia, lo que se logra potenciando los doce factores del modelo ecológico interaccional. El/la enfermero/a debe reconocer estos factores e integrarlos en su valoración, planificando su intervención para el desarrollo de resiliencia en todas las personas que dependen de su cuidado.

Los factores pertenecientes a la fuente interactiva del "yo soy", "yo estoy", son identidad, autonomía, satisfacción y pragmatismo, relacionados con fortalezas internas que se adquieren a lo largo de la vida. En ellos el/la enfermero/a puede desarrollar un plan de acción en la entrega de herramientas de solución de problemas, según el perfil de la persona o familiar que depende de su cuidado, ya sea en forma individual o grupal.

La segunda fuente interactiva, "yo tengo", está conformado por los factores vínculos, redes, modelos y metas, relacionados con el apoyo que la persona puede percibir. El/la enfermero/a puede desarrollar una importante tarea en esta área, fortaleciendo los vínculos familiares, orientando sobre las redes de apoyo, contactándolos con personas que han vivido experiencias similares que actúan como modelos en esta búsqueda de solución de problemas. Todo lo anterior se puede desarrollar a través de talleres o en forma individual, dependiendo de la condición de salud que se vivencia.

La tercera fuente interactiva, "yo puedo", incluye los factores de afectividad, autoeficacia, aprendizaje y generatividad, los que se pueden potenciar entregando las herramientas para que la persona adquiera la capacidad de creer en sus fortalezas, saliendo adelante con los recursos que posee. Al entregar un ambiente cálido y de confianza, donde la persona y/o familiar sientan seguridad y logren establecer una comunicación adecuada en busca de la estabilidad emocional y de salud, puede estimular la generación de respuestas saludables.

Ahora bien, frente a las respuestas no resilientes, es posible plantear intervenciones de enfermería que logren modificar estos comportamientos, hasta lograr el camino a transiciones y respuestas saludables. En la figura $\mathrm{N}^{\circ} 1$ se muestra la propuesta teórica que fusiona aspectos de las teorías de Meleis y Pender con resiliencia, para facilitar la práctica de enfermería. 
FIGURA 1. PROPUESTA TEÓRICA

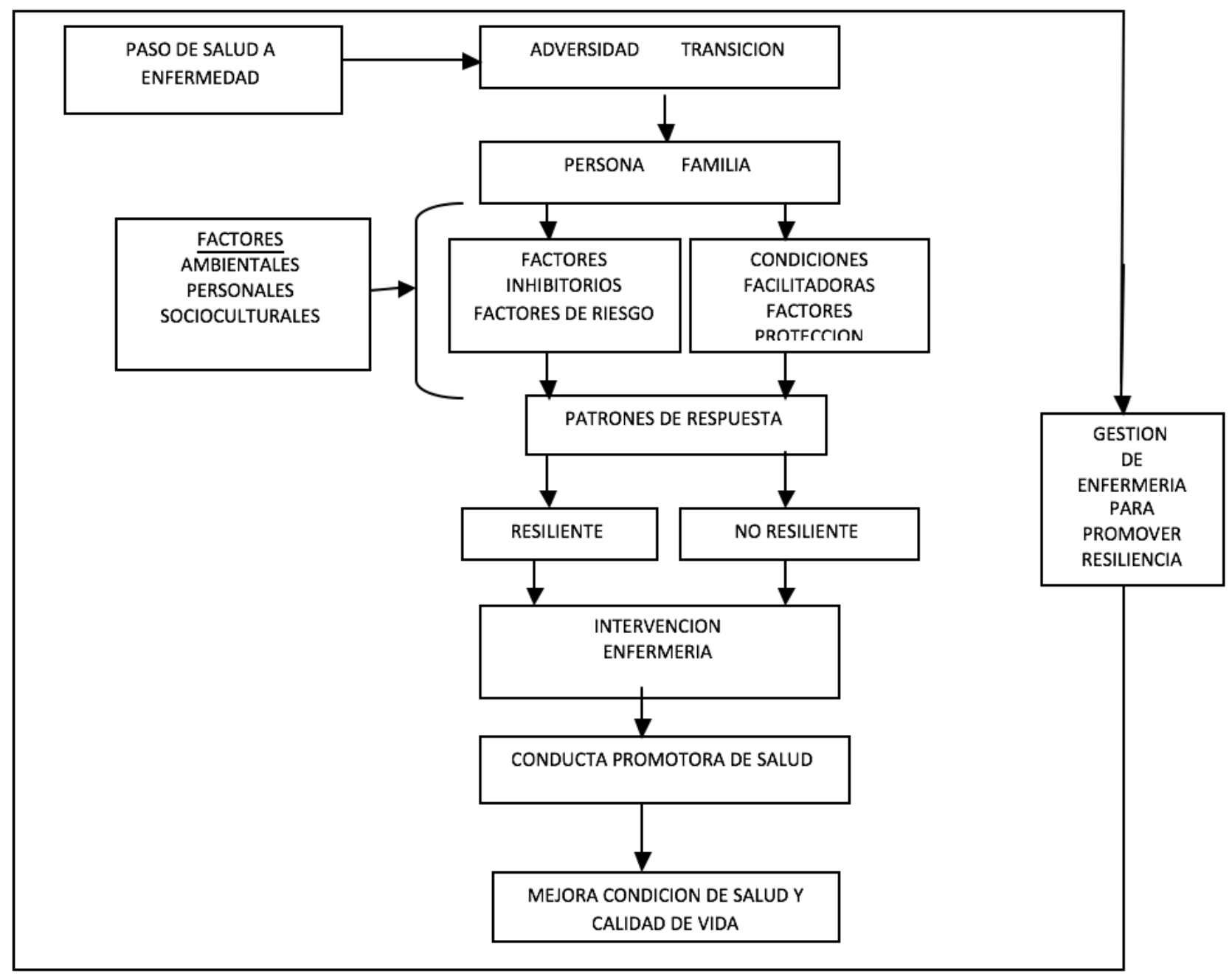

Fuente: Resiliencia en familiares de personas hospitalizadas en una Unidad de Paciente Crítico y factores relacionados (Arriagada, 2014)

\section{Consideraciones finales}

Detectar los factores amenazantes de resiliencia, ya sea en la persona y/o familiar, direcciona la intervención de enfermeros/as para obtener una mejor respuesta frente a la adversidad de las personas, transformándolos en participes de su cuidado, mejorando la adherencia al tratamiento, disminuyendo los reingresos hospitalarios y las interconsultas innecesarias, por ende, contribuyendo a respuestas positivas y saludables.
Promover la resiliencia es un llamado a involucrarse con el usuario en su completa dimensión, teniendo una visión global como ser biosicosocial, comprendiendo la realidad en que están insertos, al tiempo de utilizar estrategias de intervención para lograr transformar a la persona y/o familiar en un pilar fundamental en el proceso de estabilización del individuo frente a la adversidad. 


\section{Referencias bibliográficas}

1. Becoña E. Resiliencia: Definición, características y utilidad del concepto. Revista de Psicopatología y Psicología clínica (España). 2006; 11(3):125-46.

2. Ospina $\mathrm{D}$, Jaramillo $\mathrm{D}$, Uribe $\mathrm{T}$. La resiliencia en la promoción de la salud de las mujeres Invest Educ Enferm. 2005; 1(23):78-89

3. Cortés J. Resiliencia: Una mirada desde la Enfermería. Cienc Enferm. 2010; 16(3): 27-32.

4. Grau C. Fomentar la resiliencia en familias con enfermedades crónicas pediátricas. Revista Española de Discapacidad. 2013; 1(1): 195-212.

5. Saavedra E, Castro A. Construcción y validación de una escala de Resiliencia para escolares. [Internet]. Universidad Católica del Maule. Chile 2010.http:/ / www.rediberoamericanadetrabajoconfamilias.org/ponentes/pdf/chi_castroriosana. pdf

6. Zhong S. Resiliencia: Propuesta y desarrollo de la definición y del marco conceptual en relación a los desastres en el ámbito sanitario. Emergencia (España) 2014; 26(2):69-77.

7. Rodríguez MV. Familia Escuela. Resiliencia Familiar. Buenos Aires (Argentina): Editorial Dunken; 2009. 168 p.

8. Gómez E, Kotliarenco M. Resiliencia familiar, un enfoque de investigación e intervención con familias multiproblemáticas. Revista de Psicología. 2010; 19(2):103-32.

9. Ludgleydson F, Teva I, Bermúdez M. Resiliencia en adultos: una revisión teórica. Centro de Investigación Mente, Cerebro y Comportamiento. Ter psicol. 2015; 33(3):257-76.

10. Andrade M, Pereira S. Resiliencia Familiar:
Nuevas perspectivas en la promoción y prevención de salud. Divers Perspect Psicol. 2011;7(1):4355.

11. Quiceno JM, Vinaccia S. Resiliencia: una perspectiva desde la enfermedad crónica en población adulta. Pensamiento Psicológico. 2011; 9(17): 69-82.

12. Puig G, Rubio JL. Manual de Resiliencia aplicada. Barcelona (España): Editorial Gedisa. 2012. $287 \mathrm{p}$.

13. Saavedra E, Villalta M. Escala de Resiliencia SV_RES para jóvenes y adultos. 2da ed. Santiago de Chile: CEANIM. 2008. 105 p.

14. Saavedra E, Castro A, Inostroza A. Niveles de resiliencia en adultos diagnosticados con y sin depresión. Revista Pequén. 2012; 2(1):161-84.

15. Marriner A, Alligood MR. Modelos y teorías de enfermería. 6ta ed. España: Elsevier; 2007. 850 p.

16. Osorio G, Rosero M, Ladino A, Garcés C, Rodríguez S. La promoción de la salud como estrategia para el fomento de estilos de vida saludables. Hacia Promoc Salud. 2010 (ene-jun); 15(1):128-43.

17. Trejo F. Aplicación del modelo de Nola Pender a un adolescente con sedentarismo. Enf Neurol. 2010; 9(1):39-44.

18. Aristizábal G, Blanco D, Sánchez A, Ostiguín R. El modelo de promoción de la salud de Nola Pender. Una reflexión en torno a su comprensión. Enferm Univ.2011; 8(8):16-23.

19. Lara-Esquivel E, Torres Y, Moreno M. Actitudes y hábitos ante la prevención. Rev Enferm Inst Mex Seguro Soc. 2012; 20(1):11-7.

20. Bravo, A. El acto de cuidado de enfermería como fundamentación del quehacer profesional e 
investigativo. Av Enferm. 2016; 33(3):412-9.

21. Solé $M$, Chirveches $E$, Alsina A, Puigoriol E, Oriol $M$, Subirana $M$. La continuidad de cuidados desde el hospital de agudos: resultados. Enferm Clín. 2015; 25(4):177185.

22. Quiceno J, Vinaccia S. Resiliencia, percepción de enfermedad, creencia y afrontamiento espiritual religioso y calidad de vida relacionada con la salud en pacientes con diagnóstico de artritis reumatoide. Psicología desde el Caribe. 2013; 30(3): 590-619.

23. Ávila MM, Moral de la Rubia J. Predictores de respuestas psicológicas de duelo ante la pérdida de la salud. Revista Internacional de Psicología. 2016; 14(2):3-38. 